\title{
FURTHER DEVELOPMENTS IN ON-LINE COMPUTING AND RADIOCARBON DATING AT THE BRITISH MUSEUM
}

\author{
A D HEWSON* and J A HALL
}

Research Laboratory, The British Museum, London WC1B 3DG

\begin{abstract}
In November 1973 the British Museum Research Laboratory acquired a Hewlett Packard 2100A mini-computer for the storage, calculation and retrieval of scientific measurements made on museum objects. A part of the computer's work is the calculation of radiocarbon dates based on the liquid scintillation counting of ${ }^{14} \mathrm{C}$ activities. A system of programs and files has been developed and has been in daily use since August 1974 (Hall and Hewson, 1977).

This paper describes changes and improvements to the system to make it more flexible so that it now provides the full range of facilities required by an active ${ }^{14} \mathrm{C}$ laboratory. The reporting procedures in particular have been restructured in the light of experience. The paper will be of interest to all laboratories that have, or hope to have, access to similar mini- or micro-computers.
\end{abstract}

\section{Hardware}

At present, the computer system consists of a Hewlett Packard 21MX series E central processor containing $128 \mathrm{~K}$ 16-bit words with a $420 \mathrm{~ns}$ cycle time and direct memory access. Peripheral devices include two fixed and two exchangeable 2.4Mbyte discs, two magnetic tape drives, a paper tape reader, a paper tape punch, and an upper case printer. It is hoped that additional disc capacity will be installed in the near future.

The computer is linked, either directly or through an asynchronous multiplexer, to eight printing terminals and visual display terminals and several items of apparatus in various parts of the Research Laboratory. The radiocarbon laboratory in particular has two liquid scintillation counters (a Packard 3255 and a Packard 3315) and a visual display terminal made by Newbury Laboratories, all of which are interfaced to the central processor through the multiplexer.

The output from each liquid scintillation counter is normally relayed to both the computer and to a dedicated teletype. In this way, a copy on paper tape is maintained for a few days of all measurements of ${ }^{14} \mathrm{C}$ activity. Copies are also made from time to time of the relevant magnetic tape and disc files. There is also a facility for using one of the teletypes as an ordinary computer terminal to provide hard copy and, thus, supplement the material which may be viewed on the visual display terminal.

\section{Software}

The computer operating system, RTE IV, permits real-time multiprogramming operations and allows instructions to be relayed to programs either directly from a terminal or sequentially via a job stream. Languages used include Fortran IV, HP Algol (a subset of Algol 60), Basic, and Assembler. Programs compete for the resources of the machine through a priority system and this has been taken into account in the design of the ${ }^{14} \mathrm{C}$ system.

* Present address: Institute of Hydrology, Crowmarsh Gifford, Wallingford, Oxfordshire OX108BB 
The software for the ${ }^{14} \mathrm{C}$ system consists of three files for storing information and a number of programs. The main operating file, $C 14 W F$, is kept on one of the fixed discs and is, therefore, always available. It contains the information on all samples which are "current" $i e$, those samples for which a position in the sample-changing belt of one of the counters is presently assigned. Within the file for each sample a fixed length record plus overflow records, if necessary, contains the result of every count made on the sample in the order of measurement plus the time and date on which the measurement was made. The quench correction factor, fractionation correction factor, and the sample weight are also stored in the record and certain specified positions are used for flags and pointers so that the information in the file is correctly linked together.

When the measurements on a sample are complete, its position in the counter may be reassigned to another sample. All the information on the original sample is then moved to the second file, $C 14 A R F$, which is on a magnetic tape. In this way, valuable disc space is released but a permanent record is maintained of all the measurements.

The third file, $C 14 D F$, is a small directory file which is permanently available on disc. It contains the name of every sample with a reference to the position of the sample in the belt of the counter if it is a current sample or, if it is dormant, the point where the information on it is kept in $C 14 A R F$.

There are four main programs in the system. The first is $C 14 T$ and its sole function is to accept a reading as it is output by one of the counters and note the time at which it appears. In order to ensure that readings are always captured when they appear, no matter what the load on the computer, C14T has a very high priority and resides permanently in the computer memory. It has, therefore, been made as short as possible. The reading and time are passed to the second program, C14M, which, using the position number and counter from which the result has come, stores it in the appropriate record of $C 14 \mathrm{WF}$.

In addition, $C 14 M$ provides the facilities by which the system is controlled by the laboratory staff. The program can be run from any terminal and several different functions are available to the operator, each of which can be selected by entering the appropriate two-letter code in response to the "FN?" query of the program. Once a function has been entered, reference can be made to any current sample by following an appropriate path through a sequence of questions and responses. A mechanism is also available for restoring the information on dormant samples, thus providing access to the data on all the samples known to the system.

The functions presently available are listed in table 1 with an outline of their meaning. Several alterations have been made to the original set of commands (Hall and Hewson, 1977). Originally, C14T was timed to run just before a reading was due from a counter but improvements to the hardware have now made this unnecessary. C14T now waits continuously for readings and so the OF and GO commands 
to $C 14 M$, which controlled the time at which $C 14 T$ ran, have been withdrawn. It was also occasionally necessary to change the quench correction factor for a sample independently of the automatic sequence by which the factors are calculated, and so the IQ command was introduced.

As was explained above, when a position is reassigned, the information on the sample that previously occupied the position is transferred to a file on magnetic tape. This can be a lengthy process, and so, it is not performed immediately. Instead, C14M schedules a third program, $C 14 A R$, to do the task and continues the question and response sequence with the operator. Similarly, the calculation of age estimates (or reports) can be time-consuming; therefore, the original system $C 14 A R$ was scheduled to undertake this task, too. Recently, a completely new program, $C 14 R P$, has been written to calculate age estimates and the remainder of this paper is devoted to a description of the facilities that it provides.

\section{The new report program}

The purchase of a second liquid scintillation counter made it necessary to alter some of the software in the original ${ }^{14} \mathrm{C}$ system. This proved to be a straightforward task for $C 14 T$ and $C 14 M$ and their associated files but would have entailed major revision of the report procedures of $C 14 A R$. Experience with $C 14 A R$ revealed many areas where the functions of the program could be extended and use could be made of the new facilities provided on the computer since its initial installation. With these facts in mind, a list of objectives for the new program was drawn up as follows:

1. The program should be controlled by a group of short but explicit commands either directly from a terminal or via the job stream.

2. As an alternative to the full report procedure in which age estimates are calculated, the program should also, on command, summarize the data on a sample so that the $\mathrm{cpm} / \mathrm{g}$ of carbon (cpmpg) can be estimated.

3. It should be possible to set a time interval from which the results for a particular summary or report should be drawn, enabling straightforward, week by week, monitoring of sample activities to take place.

4. It should be possible to select particular background and modern samples against which an age estimate is to be calculated.

TABLE 1

The commands for $C 14 M$ and their meaning

\begin{tabular}{lll}
\hline & Command & \multicolumn{1}{c}{ Meaning } \\
\hline QU & (Quench) & Initiate the quench correction sequence \\
CH & (Check) & Display benzene weight, etc, of a named sample \\
SR & (Show readings) & Display the readings and times of a named sample \\
SI & (Store input) & Store input data from the paper tape back-up \\
RA & (Reassign) & Reassign a counter position to a named sample \\
RP & (Report) & Report the present age estimate of a named sample \\
FR & (Fractionation) & Store the fractionation correction factor of a named sample \\
IQ & (Insert quench) & Store the quench correction factor of a named sample \\
EX & (Exit) & Exit from the program \\
\hline
\end{tabular}


5. Routine tests should be made for significant variation amongst background and modern samples.

6. The program should generate short reports intended to give a day-to-day guide as to the progress of samples in addition to the final calculated age estimate.

7. Facilities should exist for the fractionation effect to be corrected to other than $-25 \%$ (see Stuiver and Polach, 1977) and for age corrections to be made to calculated delta values (Broecker and Olsen, 1959; 1961).

8. The program should, on command, transfer activity measurements to other computer files to facilitate more complex statistical analysis if the need arises.

9. All measurements should be monitored for evidence of radon decay.

10. Facilities should be provided for the calibration of age estimates.

The present version of $C 14 R P$ fulfills all these objectives except 9 and 10. It is written in Fortran IV using many sub-routines, several of which have been written specifically for the program. The major subroutines and their responsibilities are listed in table 2. The program has access to a main and a subsidiary output file on which to write the results of calculations. Alternatively, the output can be directed to the operator's terminal. The operator directs the programming by stringing together all or some of the commands listed in table 3 . Two of the commands, PL and DL, are complete in themselves and should logically be a part of the facilities of $C 14 M$, but it proved to be more convenient to add them to $C 14 R P$.

Five of the remaining commands, FR, DE, BG, MO, and RE, are used to over-ride the standard options which apply in most situations. Thus, FR and DE allow for unusual fractionation and age correction (see 7 , above). Certain sample names are recognized by the program as referring to reference material (OX for oxalic, SU for sucrose, etc). Normally, the program selects all such reference samples that are currently assigned a position in the relevant counter and uses them in the age calculation, but by using the BG and MO commands, the selection of reference samples can be altered. Finally, the RE command can be used

TABLE 2

The principal sub-routines of $C 14 R P$ and their duties

\begin{tabular}{|c|c|}
\hline Sub-routine & Duties \\
\hline C14DO & $\begin{array}{l}\text { The command interpreter. It sets the program on to the right path } \\
\text { to execute a command }\end{array}$ \\
\hline C14OU & Responsible for the organization of the output \\
\hline C14RE & Reads the correct material from $C 14 W F$ \\
\hline C14NA & $\begin{array}{l}\text { Ensures that samples with special names, eg, oxalic acid, are dealt } \\
\text { with correctly }\end{array}$ \\
\hline C14FE & Fetches readings on a sample in accordance with the $\mathbf{R E}$ command \\
\hline C14SJ & Computes the summary statistics \\
\hline C14ST & Manipulates and writes the data supplied by C14SJ and C14RE \\
\hline $\mathrm{Cl4CH}$ & Performs chi-square tests of homogeneity \\
\hline
\end{tabular}


to limit the selection of measurements for summaries or reports to a given number or to a given time interval within which the measurements took place.

When the operator calls for a summary of the results for a named sample, the program first retrieves the counting measurements for that sample within the constraints of any previous RE command and transfers thern to the subsidiary output file. They are then available to be read by other programs (see 8 , above). The program then calculates and writes on the main output file the mean, standard error, and poisson error of the results and also the factor (or multiplier) by which the net count rate must be multiplied to yield the estimate of the cpmpg of the sample. Control then returns to the operator.

The report sequence commences in a similar fashion to the summary sequence but the transfer of results to the subsidiary file is omitted. Instead, the subsidiary file is used later in the report sequence to hold brief details of the age estimates (see 6, above). An RE command is generated internally to span the time interval during which the sample was actually counted. The program summarizes the appropriate results for the background and the modern reference samples as specified by any previous BG or MO commands or, in the absence of such commands, it summarizes the results for all the current reference samples in the appropriate counter. A second RE command is generated to span a time interval reaching further into the past and the reference results collected during the interval are summarized to give the "rolling" background and "rolling" modern count rates.

At this point, the program has generated the mean, standard error, and multiplier for the sample and each modern and rolling modern plus the mean and standard error for each background and rolling background. The next step is to compare the mean background count rates using a chi-square test and print the result and the weighted mean. Three separate estimates of the standard error of the weighted mean are generated and printed. The first is the "internal" error and is computed by combining the standard errors of each background reference sample as if the true count rates are, in fact, equal. The second is the "comprehensive" error and is so called because it is an attempt to include all the errors made in radiocarbon activity measurements. It is set in a data statement at the beginning of the program and is, therefore, open to

TABLE 3

The commands for C14RP and their meaning

\begin{tabular}{lll}
\hline & \multicolumn{1}{c}{ Command } & \multicolumn{1}{c}{ Meaning } \\
\hline RP & (Report) & Calculate age estimates of named samples \\
SU & (Summary) & Calculate summary statistics of named sample \\
PL & (Position list) & List names and positions of current samples \\
DL & (Directory list) & List names and record numbers of all samples \\
RE & (Readings selection) & Select results by time or by number \\
FR & (Fractionation) & Set value to which fractionation is to be corrected \\
DE & (Delta) & Set value to which delta is to be corrected \\
BG & (Background) & Select background reference samples \\
MO & (Modern) & Select modern reference samples \\
\hline
\end{tabular}


adjustment. It is anticipated that this term will eventually become the best estimate of the measurement error. The third error estimate is the "external" error and is calculated on the assumption that the true count rates of the background samples are dissimilar. The program repeats the calculations for the rolling background results.

Subsequently, the net sample cpmpg is calculated based on both the background count rate and the rolling background count rate. Once again, three error estimates are calculated by combining the sample error with the internal, comprehensive, and external background error terms. The net modern cpmpg is calculated and printed for each modern and rolling modern reference sample, the errors being calculated in the same manner as for the sample. It should be noted that the results for the modern samples are correlated because they are based on the same estimate of the background count rate. No correction is made for this effect. The program also calculates but does not print the net rolling modern cpmpg relative to the current background estimate for each modern standard.

A chi-square test of homogeneity is performed for each set of modern cpmpg estimates using the internal error estimates. This is not strictly correct because of the correlation noted above, so that the result can only be treated as a guide. The results of the three tests, the weighted means, and the three types of error estimates are printed.

Finally, the program calculates and prints the age estimates and errors based on the current background and modern results, the current background and rolling modern results, and the rolling background and rolling modern results, using, in each case, both the old and the new ${ }^{14} \mathrm{C}$ half-lives. The results based on the old half-life are duplicated on the subsidiary file and then control returns to the operator.

Writing the program has focussed attention on the problems of deriving correct methods for estimating radiocarbon ages. A survey of recent issues of Radiocarbon reveals that few workers publish explicit details of their methods for assessing and combining measurement errors. The principle exceptions are Callow, Baker and Hassall (1965), Otlet (1976), and Pearson (1979). A detailed analysis of the methods used by this program and the reasons for their use will be given elsewhere (Hewson, $\mathrm{ms}$ in preparation).

\section{REFERENCES}

Broecker, W S and Olson, E A, 1959, Lamont radiocarbon measurements VI: Am Jour Sci, Radiocarbon Supp, v 1, p 111-132.

1961, Lamont radiocarbon measurements VIII: Radiocarbon, v 3, p 176-204.

Callow, W J, Baker, M J and Hassall, G I, 1965, National Physical Laboratory radiocarbon measurements III: Radiocarbon, v 7, p 156-161. Hall, J A, and Hewson, A D, 1977, On-line computing and radiocarbon dating at the
British Museum: Jour Archaeol Sci, v 4, p 89-94.

Otlet, R L, 1979, An assessment of laboratory errors in liquid scintillation methods of ${ }^{14} \mathrm{C}$ dating, in Berger, Rainer, and Suess, H E, eds, Radiocarbon dating, Internatl conf on radiocarbon dating, 9th, Proc: Los Angeles, Univ California Press,
p 256-267.

Pearson, G W, 1979, Precise ${ }^{14} \mathrm{C}$ measurement by liquid scintillation counting: Radiocarbon, v 21, p $1-21$ Stuiver, Minze and Polach, H A, 1977, Discussion: Reporting of ${ }^{14} \mathrm{C}$ data: Radiocarbon,
v 19, p 355-363. 